\title{
Padronização do Desmame da Ventilação Mecânica em Unidade de Terapia Intensiva: Resultados após Um Ano*
}

\author{
Standardization of Weaning of the Mechanical Ventilation \\ in a Intensive Care Unit: Results afterwards One Year
}

\author{
Luiz Rogério de Carvalho Oliveira' ${ }^{1}$ Anderson José2, Elaine Cristina Polleti Dias ${ }^{3}$, \\ Cíntia Ruggero ${ }^{4}$, Camila Viteli Molinari ${ }^{5}$, Paulo Antonio Chiavone 6.
}

\section{RESUMO}

JUSTIFICATIVA E OBJETIVOS: O desmame da ventilação mecânica é o processo de transição da ventilação mecânica para a espontânea. A prática atual do desmame mostra que o empirismo é insuficiente e inadequado. Em contrapartida, as padronizações de desmame proporcionam melhor condução no processo. $\mathrm{O}$ objetivo deste estudo foi avaliar os efeitos da aplicação de um protocolo de desmame da ventilação mecânica em uma unidade de terapia intensiva.

1. Fisioterapeuta Especialista em Fisioterapia em Pneumologia e Fisioterapia Respiratória, Supervisor do Curso de Fisioterapia Pneumo-Funcional e Fisioterapia Intensiva da Santa Casa de São Paulo, Fisioterapeuta da UTI do Hospital Paulistano.

2. Fisioterapeuta Especialista em Fisioterapia em Pneumologia e Fisioterapia Respiratória, Supervisor do Curso de Fisioterapia Pneumo-Funcional e Fisioterapia Intensiva da Santa Casa de São Paulo, Professor do Curso de Fisioterapia da Universidade Nove de Julho (UNINOVE).

3. Fisioterapeuta Especialista em Fisioterapia Cardiorrespiratória, Supervisora do Curso de Fisioterapia Pneumo-Funcional e Fisioterapia Intensiva da Santa Casa de São Paulo.

4. Fisioterapeuta do Hospital de Vila Alpina.

5. Fisioterapeuta, Especialista em Fisioterapia Pneumo-funcional, Supervisora do Curso de Fisioterapia Pneumo-Funcional da Santa Casa de São Paulo.

6. Doutor em Medicina, Professor Assistente da Faculdade de Ciências Médicas da Santa Casa de São Paulo, Diretor do Serviço de Terapia Intensiva da Santa Casa de Paulo

*Recebido da Instituição: Irmandade da Santa Casa de Misericórdia de São Paulo - Serviço de Terapia Intensiva

Apresentado em 09 de março de 2006

Aceito para publicação em 05 de junho de 2006

Endereço para correspondência:

Luiz Rogério de Carvalho Oliveira

Rua lbiratinga, 319

02468-040 São Paulo, SP

Fone: (11) 62393007 ou 98006175

E-mail: luizrogerio_oliveira@msn.com

(C)Associação de Medicina Intensiva Brasileira, 2006
MÉTODO: Foram incluídos no estudo todos os pacientes em programa de liberação da ventilação mecânica, em que se acompanhou a evolução do desmame com utilização de um protocolo pré-estabelecido pelo serviço já publicado.

RESULTADOS: Foram estudados 127 pacientes. Houve sucesso no desmame em $91 \%$ (115) e insucesso em $9 \%$ (12). A ventilação não-invasiva após a extubação foi utilizada em 19\% (24) deles. Nenhum óbito foi observado. Comparando-se o grupo de pacientes em que houve sucesso com o grupo em que houve falha, não foi encontrada diferença estatística significativa quanto ao sexo $(p=0,96)$, APACHE II (19,5 versus $18,6 p=$ $0,75)$, risco de óbito ( $29 \%$ versus $22 \% p=0,54)$, Pimáx (38 versus $\left.32 \mathrm{cmH}_{2} \mathrm{O} p=0,17\right)$, tempo de ventilação mecânica (6 versus 7 dias $p=0,70$ ), relação $\mathrm{PaO}_{2} / \mathrm{FiO}_{2}$ (324 versus $312 p=0,83$ ), modalidade de desmame (PSV ou Tubo T p = 0,29). Foram encontradas diferenças significativas no valor de índice de respiração rápida superficial (IRRS) (59 versus $77 p=0,02)$ e no tempo de desmame ( 1 versus 30 horas $p<0,001$ ).

CONCLUSÕES: O desmame da ventilação realizado seguindo a padronização trouxe melhora na sua condução, mantendo o alto índice de sucesso com baixa mortalidade.

Unitermos: desmame, protocolo, ventilação mecânica

\section{SUMMARY}

BACKGROUND AND OBJECTIVES: The weaning of mechanical ventilation is the process of transition from mechanical ventilation to spontaneous. The actual practice weaning reveals that the empirism is insufficient and inadequate. On the other side, the standardization of the weaning provides best conductions in the process. The objective of this study was to evaluate the effects of the application of a weaning protocol in an intensive care unit. 
METHODS: Method: It was included in this study all the patients in program of liberation from the mechanical ventilation, in which the evolution of the weaning was followed by the utilization of a pre-establish protocol and publicated by the service.

RESULTS: It was studied 127 patients. In 91\% (115) of the patients we had success in the weaning and unsuccess in $9 \%$ (12). The non-invasive ventilation after the extubation was utilized in $19 \%$ (24) of them. None obit was observed. Comparing the group of the patients that had success with the failed group, there were no statistically significant variations observed in sex $(p=$ $0.96)$, APACHE II (19.5 versus $18.6 p=0.75$ ), risk of obit (29\% versus $22 \% \mathrm{p}=0.54$ ), MIP (38 versus $32 \mathrm{cmH}_{2} \mathrm{O}$ $\mathrm{p}=0.17$ ), duration of mechanical ventilation (6 versus 7 days $\mathrm{p}=0.70$ ), $\mathrm{PaO}_{2} / \mathrm{FiO}_{2}$ relation (324 versus $312 \mathrm{p}$ $=0.83$ ), weaning modality (PSV or T Tube $p=0.29$ ). There were statistically significant variations observed in rapid shallow respiratory index (59 versus $77 \mathrm{p}=$ 0.02 ) and duration of the weaning (1 versus 30 hours $\mathrm{p}<0.001$ ).

CONCLUSIONS: The weaning of the ventilation realized following the standardization brought improvement in its conduction, maintaining the high success index with low mortality.

Key Words: mechanical ventilation, standardization, weaning

\section{INTRODUÇÃO}

Apesar de ser uma intervenção importante no paciente com insuficiência respiratória aguda, a ventilação mecânica pode induzir diversas complicações, que podem aumentar a morbimortalidade de um paciente grave, portanto, é importante abreviar o tempo no qual o paciente está sob ventilação artificial invasiva, restabelecendo a ventilação espontânea tão logo seja possível1-6.

$\mathrm{Na}$ maioria dos pacientes, após a recuperação do evento agudo que motivou a ventilação mecânica, o retorno gradual à ventilação espontânea pode ser abreviado ${ }^{6-10}$. Cerca de $60 \%$ a $70 \%$ dos pacientes podem ser extubados após um breve teste em ventilação espontânea ${ }^{6-15}$. A dificuldade no desmame reside em cerca de $5 \%$ a $30 \%$ dos pacientes, que não conseguem ser retirados do ventilador em uma primeira ou segunda tentativa ${ }^{14,16}$.

A ventilação mecânica e o processo de sua retirada são recentes na história da Medicina. Isso faz com que atualmente essas técnicas tenham pouco embasamen- to científico que oriente as condutas dos profissionais que lidam com esta rotina ${ }^{16-21}$. O empirismo aplicado ao desmame da ventilação mecânica leva a piora na qualidade do seu processo e conseqüentemente a aumento na taxa de falha, morbidade e mortalidade. Atualmente, com o aumento dos estudos relacionados ao desmame, nota-se uma melhora na condução deste processo, tornando-o cada vez mais uma ciência e

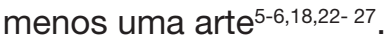

Os protocolos de desmame estudados até o momento têm apresentado alto grau de eficiência ${ }^{5-6,18,23,25,26,28}$. A aplicação de um protocolo de desmame com rigor científico e um método padronizado pode trazer várias vantagens em relação ao desmame empírico. Dentre essas vantagens destacam-se a redução significativa no tempo de desmame, redução na relação entre tempo de desmame e tempo total de ventilação mecânica, diminuição dos índices de insucessos e re-intubações, diminuição da mortalidade, menor tempo de internação na unidade de terapia intensiva e de internação hospitalar, conseqüentemente redução dos custos hospitalares s $^{7,12,14,15,18,20,22,23}$.

Há uma série de protocolos verificados em ensaios clínicos, entretanto, ainda há carência de estudos que avalie o seu desempenho em longo prazo nas unidades de terapia intensiva. Recentemente, foi publicado um ensaio clínico, onde foi desenvolvido um protocolo de desmame, comparando-o com o desmame realizado sem rigor no método. Os resultados desse estudo mostraram melhora significativa na qualidade do processo de desmame nos pacientes que seguiram o protocolo, com diminuição do tempo de ventilação mecânica e da relação entre tempo de desmame e o tempo de ventilação mecânica, redução do tempo de desmame, diminuição das taxas de insucesso e da taxa de mortalidade ${ }^{23}$. Porém, havia ainda a necessidade de avaliar o desempenho do protocolo de desmame em longo prazo, inserindo-o na prática clínica diária.

O objetivo deste estudo foi avaliar os efeitos em longo prazo, a padronização do desmame da ventilação mecânica em uma unidade de terapia intensiva, durante um ano em todos os pacientes que necessitaram de suporte ventilatório invasivo.

\section{MÉTODO}

Após a aprovação do projeto de pesquisa pelo Serviço de Terapia Intensiva e pelo Comitê de Ética em Pesquisa (CEP) da instituição, foi realizado este estudo 
prospectivo e caso-controlado no Serviço de Terapia Intensiva da Irmandade de Santa Casa de Misericórdia de São Paulo, durante o período de fevereiro de 2003 a fevereiro de 2004.

Foram incluídos no estudo todos os pacientes em ventilação mecânica, considerados aptos para o desmame de acordo com os critérios de pré-desmame descritos no protocolo (Anexo 1). Foram excluídos do estudo todos os pacientes com idade inferior a 18 anos e com traqueostomia.

Os pacientes foram divididos em dois grupos de acordo com o tempo de suporte ventilatório, no grupo A: pacientes que permaneceram sob ventilação mecânica por um período superior a 24 horas e no grupo B: pacientes que permaneceram sob ventilação mecânica por um período inferior a 24 horas, não havendo intenção em compará-los, mas somente em avaliar o desempenho em duas situações clínicas distintas.

Foi considerado desmame bem sucedido quando o paciente extubado, permanecesse sem assistência ventilatória mecânica e sem sinais de insuficiência respiratória aguda durante um período superior a 24 horas. Caso o paciente apresentasse um quadro de insuficiência respiratória aguda após a extubação, este seria submetido à ventilação mecânica não-invasiva. Foram utilizados os ventiladores mecânicos Dräger Evita $S^{\circledR}$, Dräger - Evita $2^{\circledR}$ e Dräger - Savina ${ }^{\circledR}$. A análise estatística realizada compreendeu o cálculo de médias e desvios-padrão, testes $t$ de Student, t pareado, de
Mann-Whitney e o Exato de Fisher. Foi considerada estatística significativa uma margem de erro de $5 \%$ ( $p$ $<0,05)$.

\section{RESULTADOS}

Foram estudados 127 pacientes e os resultados estão apresentados na tabela 1.

Em ambos os grupos os indicadores dos pacientes que evoluíram com sucesso no desmame em relação aos pacientes que evoluíram com falha estão na tabela 2 . Houve oito pacientes que evoluíram com extubação não planejada (extubação acidental) e a equipe decidiu não submetê-los à ventilação mecânica invasiva. Como resultados desta tentativa, três pacientes permaneceram sem necessidade de prótese endotraqueal para ventilação, permanecendo extubados, porém, nos outros cinco pacientes houve falha nesta tentativa, necessitando de re-intubação, mesmo após a aplicação de ventilação mecânica não-invasiva. Apenas um destes cinco pacientes que houve falha nesta tentativa não evoluiu para óbito durante o período de internação na UTI.

Também foram observados que 10 pacientes foram extubados sem seguir o protocolo de desmame proposto. Estes pacientes não apresentavam critérios para o desmame e para a extubação, de acordo com o protocolo. Como resultado deste processo, todos os pacientes (100\% $(n=10))$, evoluíram com falha no desmame e necessitaram de re-intubação.

Tabela 1 - Evolução do Processo de Desmame

\begin{tabular}{lccc}
\hline & Grupo A (VM > 24horas) & Grupo B (VM < 24horas) & Total 100\% \\
& $63 \%(\mathrm{n}=80)$ & $37 \%(\mathrm{n}=47)$ & $127)$ \\
\hline Sucesso & $91 \%(73)$ & $89 \%(42)$ & $91 \%(115)$ \\
Insucesso & $9 \%(7)$ & $11 \%(5)$ & $9 \%(12)$ \\
VMNI & $14 \%(10)$ & $30 \%(14)$ & $19 \%(24)$ \\
\hline
\end{tabular}

VM:ventilação mecânica; VMNI: ventilação mecânica não-invasiva.

Tabela 2 - Características do Processo de Desmame

\begin{tabular}{|c|c|c|c|c|c|c|}
\hline & \multicolumn{3}{|c|}{ Grupo A $(n=80)$} & \multicolumn{3}{|c|}{ Grupo B $(n=47)$} \\
\hline & Sucesso & Insucesso & $p$ & Sucesso & Insucesso & $p$ \\
\hline APACHE II & $20 \pm 7$ & $19 \pm 9$ & 0,753 & $15 \pm 3$ & $17 \pm 4$ & 1,0 \\
\hline Risco de Óbito (\%) & $29 \pm 20$ & $22 \pm 16$ & 0,542 & $29 \pm 5$ & $28 \pm 6$ & 0,683 \\
\hline IRRS & $59+25$ & $77+16$ & $0,022^{*}$ & $50+14$ & $83+7$ & $0,001^{*}$ \\
\hline Pimáx $\left(\mathrm{cmH}_{2} \mathrm{O}\right)$ & $-38 \pm 10$ & $-32 \pm 10$ & 0,17 & $-38 \pm 11$ & $-31 \pm 3$ & $0,001^{*}$ \\
\hline $\mathrm{PaO}_{2} / \mathrm{FiO}_{2}$ & $324 \pm 75$ & $312 \pm 46$ & 0,833 & $215 \pm 18$ & $212 \pm 21$ & 0,56 \\
\hline $\begin{array}{l}\text { Tempo de VM } \\
\text { (horas) }\end{array}$ & $150 \pm 99$ & $161 \pm 10$ & 0,703 & $8 \pm 5$ & $11 \pm 4$ & 0,11 \\
\hline
\end{tabular}

"Com valor estatístico significativo $(p<0,05)$.

APACHE II: Acute Physiology and Chronic Health Evaluation II; Pimáx: pressão inspiratória máxima; IRRS: índice de respiração rápida e superficial (f/Vt); PaO//FiO relação entre a pressão parcial de oxigênio arterial e fração inspirada de oxigênio; Tempo de VM: tempo total de ventilação mecânica. 


\section{DISCUSSÃO}

Em estudo anterior ${ }^{23}$, controlado, prospectivo e aleatório, foram comparados os resultados do desmame da ventilação mecânica utilizando um protocolo versus o desmame empírico, em pacientes que necessitaram de ventilação mecânica invasiva por mais de 24 horas. Ele mostrou taxa de falha no desmame de $65 \%$ para o desmame empírico e de apenas 5\% para o desmame padronizado. Pelos resultados animadores obtidos, foi desenvolvida esta pesquisa, utilizando o mesmo protocolo por um ano, em todos os pacientes que necessitassem de suporte ventilatório.

Neste estudo, a taxa de sucesso do desmame foi $91 \%$ para os pacientes que permaneceram sob ventilação mecânica por um período superior a 24 horas e de $89 \%$ para pacientes submetidos à ventilação mecânica menos que 24 horas, totalizando uma taxa de sucesso de $91 \%$ dos casos. Constatou-se uma taxa extremamente baixa de falha no desmame, inclusive inferiores à grande maioria de outros estudos recentemente publicados. 1,6-10,18,19,21,24-26,29-34

Estes resultados dão segurança à equipe interprofissional quanto à decisão de extubar ou não um paciente, transformando esta decisão de um julgamento pessoal para uma decisão segura e científica. A conduta da equipe, bem como o seu bom desempenho diagnóstico e terapêutico são primordiais na condução dos pacientes em processo de desmame. . $23,26-28,35,36^{-}$

Quando o desmame é bem conduzido, há uma sensível melhora na evolução do paciente e podem ocorrer repercussões positivas diretas, como por exemplo, a diminuição do tempo de desmame e da ventilação mecânica, menor índice de falhas no desmame, menor taxa de re-intubação, traqueostomia e pneumonia, diminuição do tempo de internação na unidade de terapia intensiva e hospitalar, além da redução dos custos hospitalares..$^{25,26}$

O momento adequado para submeter um paciente ao desmame é produto de parâmetros preferencialmente estáveis, dos exames clínicos diários e desempenho pulmonar ${ }^{11}$. Quando se trata de liberar do suporte ventilatório pacientes com real dificuldade de retorno à ventilação espontânea, o sucesso continua vinculado ao seu acompanhamento cuidadoso por uma equipe multidisciplinar experiente e competente, capaz de indicar ou contra-indicar um desmame com base em dados sólidos, evitando o desgaste físico e a ansiedade excessiva do paciente. Para tanto, são importantes a detecção precoce de possível falha, a avaliação de sua origem, o repouso da musculatura respiratória e a implantação de medidas terapêuticas antes de nova tentativa. ${ }^{1,12,23}$

As falhas no desmame devem ser evitadas ao máximo; um desmame mal conduzido e o seu conseqüente insucesso pode proporcionar aumento de morbidade, especialmente no que diz respeito à incidência de infecções respiratórias decorrentes principalmente de uma re-intubação, com conseqüente aumento na mortalidade. . $^{8-10,13,23}$

$\mathrm{Na}$ tentativa de se evitar estas falhas no desmame da ventilação mecânica e as suas possíveis complicações, diversos indicadores tem sido pesquisados, porém poucos possuem poder preditor satisfatório. Apesar de muito estudado, faltam evidências de que o índice de respiração rápida e superficial (IRRS) consiga realizar esta função de forma eficaz ${ }^{1,2,12,13,36}$. Este estudo demonstrou uma grande taxa de falsos-positivos apresentados por este índice e também para a pressão inspiratória máxima (Pimáx), que apesar de terem apresentado diferenças estatísticas significativas, estavam além do valor de corte que prediz a falha no desmame (IRRS < 104 e Pimáx $>-25 \mathrm{cmH}_{2}$ O) demonstrando a incapacidade destes índices em predizer com segurança uma possível falha no desmame. Estudos apontam que outros índices preditores do sucesso do desmame como capacidade vital, volume minuto, $\mathrm{PaO}_{2} / \mathrm{FiO}_{2}$, freqüência respiratória, complacência estática, resistência das vias aéreas, entre outros, mostram-se temporal e fisiologicamente incapazes de predizer falhas no desmame cujas causas sejam não respiratórias, isto é, tais índices ignoram condições não pulmonares que podem afetar a saída da ventilação mecânica ${ }^{1}$. O mesmo ocorre com os índices de oxigenação e a análise dos gases arteriais. ${ }^{37,38}$

Não houve diferença significativa entre os valores de APACHE II e o risco de óbito entre os pacientes que foram retirados do desmame com sucesso e insucesso. Também não houve óbitos durante o processo de desmame até a alta da UTI, provavelmente porque a aplicação do protocolo pôde resultar numa maior segurança na condução de um paciente crítico, mesmo em pacientes com alto risco de óbito. Em estudo pré$\mathrm{vio}^{23}$, o protocolo de desmame foi aplicado em pacientes com APACHE II de 20 pontos e risco de óbito de $31 \%$ e cursaram com um processo de desmame sem intercorrências e nenhum óbito ocorreu durante o processo até a alta da UTI.

O sucesso do desmame depende da redução da demanda e, concomitantemente, do aumento da capa- 
cidade ventilatória sugerindo então melhor reserva ventilatória ${ }^{39}$. A falha no teste de autonomia pode refletir uma baixa resistência ventilatória, a qual desenvolve-se pelo desuso desta musculatura durante a permanência em ventilação mecânica, resolução incompleta da causa da insuficiência respiratória ou desenvolvimento de novos problemas ${ }^{12}$. Em pacientes com sucessivos insucessos de desmame, o desmame gradual deve ser considerado enquanto os fatores responsáveis pela dependência ventilatória devem ser corrigidos.

A maior dificuldade na aplicação do protocolo encontra-se na correta identificação dos pacientes realmente aptos para iniciar o processo de desmame e concluir com uma extubação bem sucedida. A equipe deve observar cuidadosamente os benefícios da rápida liberação da ventilação mecânica, porém não deve ignorar os riscos da sua realização prematura. Uma vez que o paciente é considerado apto para ser retirado da ventilação mecânica, a melhor forma de identificar se ele é capaz de ventilar espontaneamente é através do teste de autonomia ventilatória, seja este em tubo-T ou PSV de $7 \mathrm{cmH}_{2} \mathrm{O}^{12-13,40}$. Porém, os critérios quanto à falha no teste devem ser observados, sabendo que critérios muito permissivos aumentam o risco de falha no desmame e re-intubação e os critérios rígidos podem manter o paciente em ventilação mecânica por um longo período e submetê-lo às complicações da ventilação mecânica.

Pelo exposto, conclui-se que o desmame da ventilação mecânica realizado seguindo uma padronização trouxe melhora na condução do desmame, mantendo alto o índice de sucesso e baixa mortalidade, porém ainda faltam critérios mais precisos para a condução do desmame em pacientes com menos de 24 horas de ventilação mecânica.

\section{Anexo 1 - Protocolo de Desmame da Ventilação Mecânica}

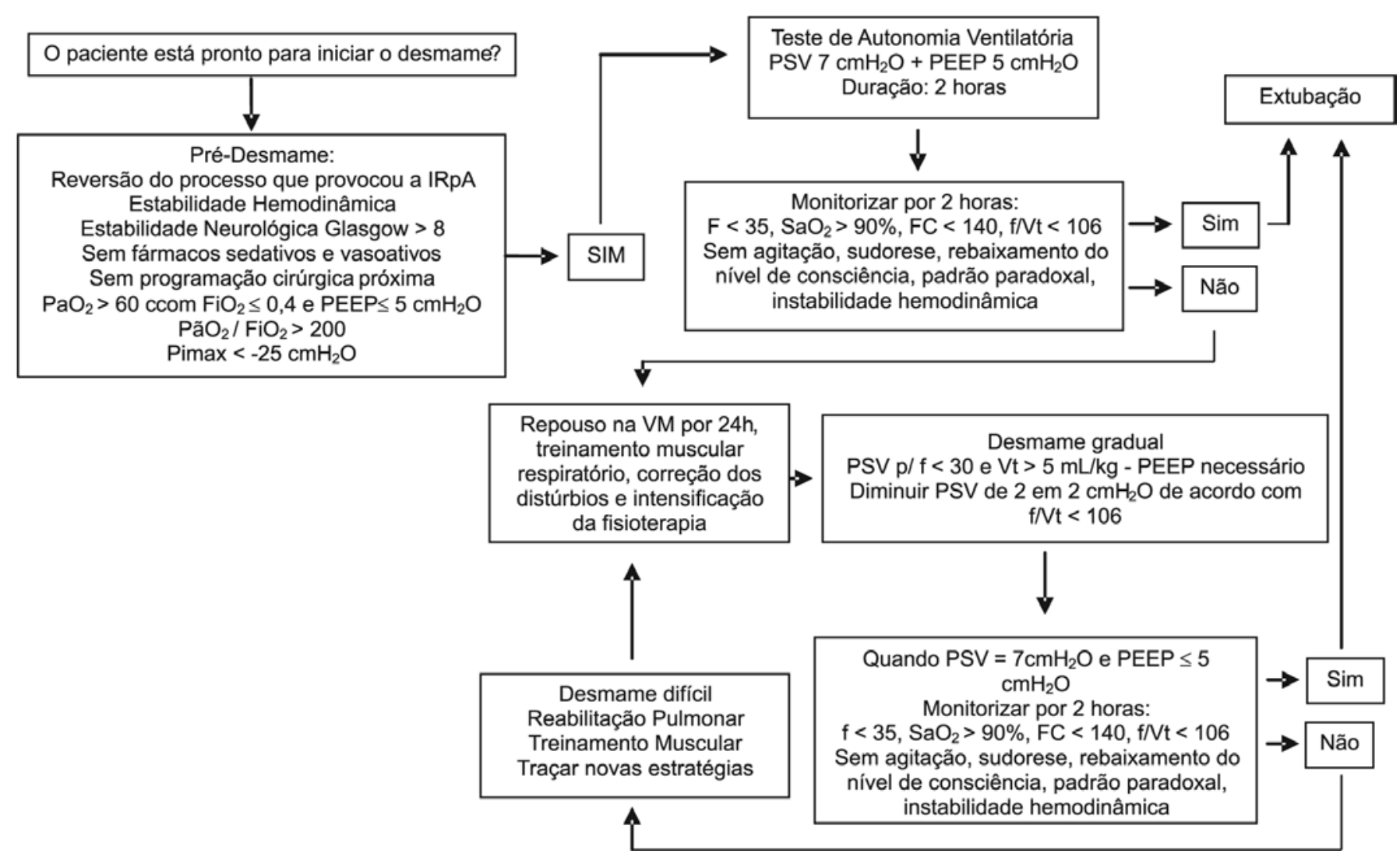




\section{REFERÊNCIAS}

01. Afessa B, Hogans L, Murphy R - Predicting 3-day or 7-day outcomes of weaning from mechanical ventilation. Chest, 1999;116:456-461.

02. Alia I, Esteban A - Weaning from mechanical ventilation. Crit Care, 2000;4:72-80.

03. Borges VC, Andrade A Jr, Lopes AC - Desmame da ventilação mecânica. Rev Bras Clin Terap, 1999;25:171-178.

04. Coplin WM, Pierson DJ, Cooley KD et al - Implications of extubation delay in brain-injured patients meeting standard weaning criteria. Am J Respir Crit Care Med, 2000;161:1530-1536.

05. Dries DJ - Weaning from mechanical ventilation. J Trauma, 1997;43:372384.

06. Ely EW, Bennett PA, Bowton DL et al - Large scale implementation of a respiratory therapist-driven protocol for ventilator weaning. Am J Respir Crit Care Med, 1999;159:439-446

07. Ely EW; Meade MO, Haponik EF et al - Mechanical ventilator weaning protocols driven by nonphysician health-care professionals: evidencebased clinical practice guidelines. Chest, 2001;120:Suppl6):454S-463S.

08. Epstein S, Ciubotaru R - Independent effects of etiology of failure and time to reintubation on outcome for patients failing extubation. Am $\mathrm{J}$ Respir Crit Care Med, 1998;158:489-493.

09. Epstein SK, Ciubotaru RL, Wong JB - Effect of failed extubation on the outcome of mechanical ventilation. Chest, 1997;112:186-192.

10. Epstein SK, Nevins ML, Chung J - Effect of unplanned extubation on outcome of mechanical ventilation. Am J Resp Crit Care Med, 2000;161:1912-1916.

11. Epstein SK - Predicting extubation failure: is it in (on) the cards? Chest, 2001;1120:1061-1063.

12. Esteban A, Alia I, Gordo F et al - Extubation outcome after spontaneous breathing trials with T-tube or pressure support ventilation. Am J Respir Crit Care Med, 1997;156:459-465.

13. Esteban A, Alia I, Tobin MJ et al - Effect of spontaneous breathing trial duration on outcome of attempts to discontinue mechanical ventilation. Am J Resp Crit Care Med, 1999;159:512-518.

14. Goldwasser R - Desmame da Ventilação Mecânica, em: Carvalho CR - Ventilação Mecânica. São Paulo: Atheneu, 2000;425-438.

15. Goldwasser R, Messeder O, Amaral JLG et al - Desmame da ventilação mecânica. J Pneumol, 2000;26:(Suppl2):S54-S60.

16. RothmanA, Barbas CSV, Bueno MAS - Desmame da Ventilação Mecânica, em: Knobel E - Condutas no Paciente Grave. $2^{a}$ Ed, São Paulo, Atheneu, 1998;1:381-391.

17. Hess D - Ventilator modes used in weaning. Chest, 2001;120:474S476S.

18. Horst HM, Mouro D, Hall-Jenssens RA, Pamukov N. - Decrease in ventilation time with a standardized weaning process.Arch Surg. 1998 May;133(5):483-8; discussion 488-9

19. Khamiees M, Raju P, DeGirolamo A et al - Predictors of extubation outcome in patients who have successfully completed a spontaneous breathing trial. Chest, 2001;120:1262-1270.

20. Knebel AR, Janson-Bjerklie SL, Malley JD et al - Comparison of breathing comfort during weaning with two ventilatory modes. Am J Respr Crit Care Med, 1994;149:14-18.

21. Sahn SA, Lakshminarayan S - Bedside criteria for discontinuation of mechanical ventilation. Chest, 1973;63:1002-1005.
22. Kupfer $\mathrm{Y}$, Tessler $\mathrm{S}$ - Weaning the difficult patient: the evolution from art to science. Chest, 2001;119:7-9.

23. Oliveira LRC, José A, Dias ECP et al - Protocolo de desmame da ventilação mecânica: efeitos da sua utilização em uma Unidade de Terapia Intensiva. Um estudo controlado, prospectivo e randomizado. RBTI, 2002;14:22-32.

24. Kollef $\mathrm{MH}$, Shapiro SD, Silver $\mathrm{P}$ et al - A randomized, controlled trial of protocol-directed versus physician-directed weaning from mechanical ventilation. Crit Care Med, 1997;25:567-574.

25. Saura P, Blanch L, Mestre J et al - Clinical consequences of the implementation of a weaning protocol. Intensive Care Med, 1996;22:1052-1056.

26. Scheinhorn DJ, Chao DC, Stearn-Hassenpflug $M$ et al - Outcomes in post-ICU mechanical ventilation: a therapist-implemented weaning protocol. Chest, 2001;119:236-242.

27. Wood G, MacLeod B, Moffatt S - Weaning from mechanical ventilation: physician-directed vs a respiratory therapist-directed protocol. Respir Care, 1995;40:219-224.

28. Straus C, Louis B, Isabey D et al - Contribution of the endotracheal tube and the upper airway to breathing workload. Am J Respir Crit Care Med, 1998;157:23-30.

29. Meade M, Guyatt G, Cook D et al - Predicting success in weaning from mechanical ventilation. Chest, 2001;120:(Suppl6):400S-424S.

30. Esen F, Denkel T, Telci L et al - Comparison of pressure support ventilations (PSV) and intermittent mandatory ventilation (IMV) during weaning in patients with acute respiratory failure. Adv Exp Med Biol, 1992;317:371-376.

31. Brochard L, Rauss A, Benito S et al - Comparison of three methods of gradual withdrawal from ventilatory support during weaning from mechanical ventilation Am J Respir Crit Care Med, 1994;150:896-903.

32. Esteban A, Frutos F, Tobin MJ et al - A comparison of four methods of weaning patients from mechanical ventilation. $\mathrm{N}$ Engl $\mathrm{J}$ Med, 1995;332:345-350.

33. Capdevila XJ, Perrigault PF, Perey PJ et al - Occlusion pressure and its ratio to maximum inspiratory pressure are useful predictors for successful extubation following T-piece weaning trial. Chest, 1995;108:482-489.

34. Goodnough SK - Multidimensional predictors of success or failure with early weaning from mechanical ventilation after cardiac surgery. Nurse Res, 1994;43:4-10.

35. Maclntyre NR - Evidence-based guidelines for weaning and discontinuing ventilatory support. Chest, 2001;120:375S-396S.

36. Meade M, Guyatt G, Sinuff T - Trials comparing alternative weaning modes and discontinuation assessments. Chest, 2001;120:(Suppl6):425S437S.

37. Salam A, Smina M, Gada $P$ et al - The effect of arterial blood gas values on extubation decisions. Respir Care, 2003;48:1033-1037.

38. José A, Dias ECP, Santos VLA et al - Valor preditivo dos gases arteriais e índices de oxigenação no desmame da ventilação mecânica. RBTI, 2001;13:50-57.

39. Vitacca M, Ambrosino N, Clini E et al - Physiological response to pressure support ventilation delivered before and after extubation in patients not capable of totally spontaneous autonomous breathing. Am J Resp Crit Care Med, 2001;164:638-641.

40. Vallverdu I, Calaf N, Subirana $M$ et al - Clinical characteristics, respiratory functional parameters, and outcome of a two-hour T-piece trial in patients weaning from mechanical ventilation. Am J Resp Crit Care Med, 1998;158:1855-1862. 